\title{
$\mathrm{JJ}-4$
}

\section{Evaluation of Mental Stress Using Wireless Patch-style Heart Activity Monitoring System}

\author{
Heui Kyung Yang ${ }^{1}$, Jeong Whan Lee ${ }^{2}$, Kang Hwi Lee ${ }^{2}$, Young Jae Lee ${ }^{2}$, \\ Hee Jung $\mathrm{Choi}^{3}$, Dong Jun Kim ${ }^{1}$ \\ ${ }^{1}$ School of Electronics and Information Engineering, CheongJu University, CheongJu, Korea \\ ${ }^{2}$ School of Biomedical Engineering, KonKuk University, ChungJu, Korea \\ ${ }^{3}$ Department of Nursing, KonKuk University, ChungJu, Korea \\ hkyang@,cju.ac.kr
}

\begin{abstract}
For evaluation of mental stress, this research gives out the questionnaire and measures the number of hormone through blood test. The activity of autonomic nervous system was evaluated through the analysis of ECG signal measured with wireless patch-style heart monitoring system which is able to measure and record the data.

Psychological evaluation was performed for measuring mental stress by questionnaire research. The result of physiological evaluation was acquired through hormone test. Various parameters of frequency and time domain which are relative to psychological and physiological evaluation were extracted from heart rate variability.
\end{abstract}

Keywords: wireless patch-style heart monitoring system, mental stress, questionnaire, blood test, heart rate variability

\section{Introduction}

The wireless patch-style heart activity monitoring system (HAMS) which was used for recording ECG signal in this study is self-developed. In the same subjects who were exposed under stress and non-stress, the questionnaire was given out, the amount of the stress hormone was measured by blood test and the ECG signal was recorded. Through the analysis of ECG signal which is measured with wireless ECG module, the parameter highly related with mental stress[1][2] were extracted from frequency and time domain[3][4]. These parameters were certified as the meaningful factor after correlation analysis on the results from questionnaire and stress hormone test.

\section{Wireless patch-style heart activity monitoring system (HAMS)}

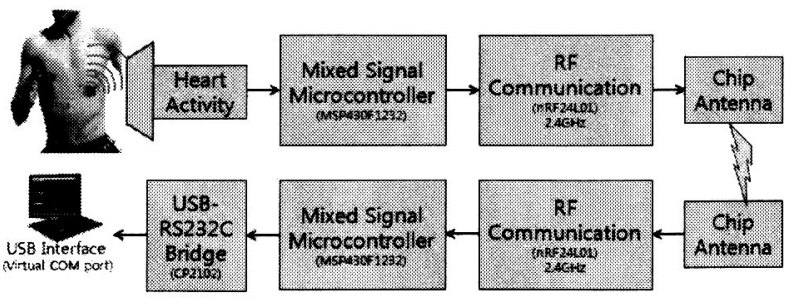

Fig. 1 The structure of HAMS

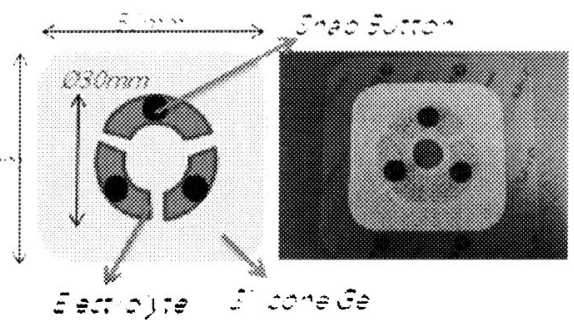

(a) patch-style electrode

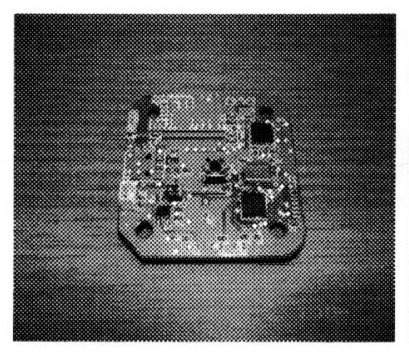

(b) transmitter

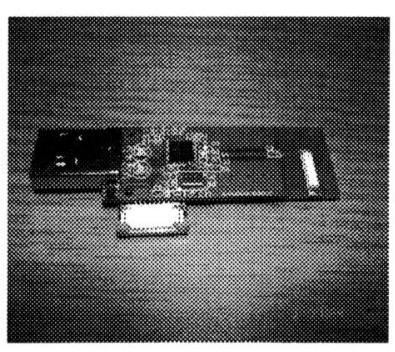

(c) receiver
Fig. 2 HAMS module

The ECG signal expresses the electrical activities of the heart. In this study, ECG signal was measured with the small size patch-style electrode which consists of three electrodes based on Einthoven's leads and is able to attach in breast. The merit of the HAMS is that it can easily measure and record the heart activity of the subject in long term. 


\section{Experiment}

This study is for stress evaluation in Nov 2006. Those subjects for this study are 41 students from department of nursing in Konkuk University. Also the experiment was done under both condition stressed and non-stressed on the same subjects. After blood test for measuring stress hormone, ECG signal was recorded for about 10 minutes in $125 \mathrm{~Hz}$ sampling frequency by using self-invented wireless ECG module. Then, the questionnaire was given out for checking about uneasiness (20-80 scales) and stress (0-100 scales).

\section{Analysis}

R-peak was detected from ECG signal measured by HAMS. And it was analyzed for 5 minutes by using HRV Analysis Software ver1.1 developed at University of Kuopio in Finland. For stress evaluation, mean RRI[s], mean Heart Rate[1/min], RMSSD[ms], NN50[count], pNN50[\%], RR tri index, TINN[ms], CSI and CVI were analyzed in time domain. And, LF $(0.04-0.15 \mathrm{~Hz}), \mathrm{HF}(0.15-0.4 \mathrm{~Hz})$ and $\mathrm{LF} / \mathrm{HF}$ ratio were analyzed in frequency domain.

The parameters from the amount of stress hormone in blood, result of questionnaire and ECG signal were compared in both conditions under the case of stress and non-stress. These parameters were certified as the meaningful factor after correlation analysis on the results from questionnaire and stress hormone test.

\section{Result}

The parameters from the amount of stress hormone (physiological factor) in blood, result of questionnaire (psychological factors, uneasiness and stress) and ECG signal were compared in both conditions under the case of stress and non-stress. As the result of this comparing and analysis, the all parameters had significant differences except CVI, LF and HF.

After correlation analysis on the results from questionnaire and stress hormone test, only several parameters in CVI, LF, $\mathrm{HF}$ and $\mathrm{LF} / \mathrm{HF}$ ratio were correlated, but the all parameters except those were highly correlated.

\section{Conclusion}

The wireless patch-style heart activity monitoring system (HAMS) which was used for recording ECG signal in this study is self-developed. It has also portability which people can measure in any place and occasion. In addition, it reduces the discomfort of subjects against existed methods. As ECG signal was measured by this module, there was remarkable difference between under case of non stress and stress.

It is expected that the HAMS will be applied to health monitoring system in the future because of its convenience for measuring.

\section{References}

[1] Taketsu H., Munakata M., Ozeki O., Yokoyama K., Watanabe Y. and Takata K. : An Evaluation of the Quantitative Relationship between the Subjective Stress value and Heart Rate Variability, T. IEEE Japan, Vol.120-C, No. 1:104-110, 2000

[2] Murata A. : Measurement of mental workload by heart rate variability indexes, 人間工学 Vol.28, No.2:91-98, 1992

[3] Task Force of The European Society of Cardiology and The North American Society of Pacing and Electrophysiology: Heart rate variability-Standards of measurement, physiological interpretation, and clinical use, European Heart Journal 17:354-381, 1996

[4] Toichi M., Kubota Y., Murai T., Kamio Y., Sakihama M., Toriuchi T., Inakuma T., Sengoku A. and Miyoshi K.: The influence of psychotic states on the autonomic nervous system in schizophrenia, International J. of Psychophysiology 31:147-154, 1999 